\title{
Absorption Coefficient Measurement Advanced Method of EUV Resist by Direct-Resist Coating on a Photodiode
}

\author{
Shota Niihara*, Daiki Mamezaki, Masanori Watanabe, Tetsuo Harada, and Takeo Watanabe
}

\author{
Center for EUV Lithography, LASTI, University of Hyogo \\ 1-1-2, Koto, Kamigori-cho, Ako-gun, Hyogo, 678-1205, Japan \\ *sniihara@lasti.u-hyogo.ac.jp
}

\begin{abstract}
Since the resist thickness in EUV lithography of which base material mainly consists of an organic material is thin, the EUV photon energy is not be used efficiently for the EUV chemical reaction. In order to increase chemical reaction incidence in the EUV photon energy, a resist having a high-absorption material compounds for the EUV photons has been developed. It has been studied to increase the absorption including high-absorption materials such as hafnium and zinc, tin oxide, tellurium. For the development of the next-generation high sensitive resist materials, since it is significant to measure the EUV absorption coefficient accurately, it is necessary to measure the transmittance and resist thickness to obtain the absorption coefficient accurately. Thus, we have developed to evaluate the absorption coefficient of the EUV resist at BL 10 beamline of NewSUBARU synchrotron hlight facility. In the previous paper, we measured the EUV resist transmittance on a freestanding membrane. However, since it was very difficult to coat resist on a membrane with high uniformity, the transmittance could not be measured accurately. Thus, we have developed the precise transmittance measurement method by coating resist on a photodiode directly, and the resist thickness on a photodiode was measured by XRR accurately instead on conventional method. Finally, the accurate measurement of EUV resist absorption coefficient was achieved.
\end{abstract}

Keywords: EUV resist, Absorption coefficient, Transmittance measurement, XRR

\section{Introduction}

The extreme-ultraviolet (EUV) lithography [1] is a promising lithographic technology for high volume manufacturing of the semiconductor electronic devices. One critical issue in the EUV lithography for high volume manufacturing is development of EUV resists which has the high resolution, high sensitivity and low line-width roughness, and low outgassing, simultaneously [2,3]. Especially, the high sensitivity is strongly required to relax the specification of EUV power and maintain the highlithographic throughput. The small resist feature size requires thin resist thickness. For example, the EUV resist thickness was $50 \mathrm{~nm}$ and below at the latest EUV exposure tool [4,5]. Recently the resist thickness becomes to be $20-30 \mathrm{~nm}$ in $7 \mathrm{~nm}$ node. The caluculated transmittance of organic resist (ex. PMMA resist) will be $>85 \%$ with a thickness of 30 $\mathrm{nm}$. Since the EUV photon energy is not react efficiently with an organic resist material because of having high transmittance, increasing the resist sensitivity is not so easy. In order to achieve high sensitive resist, some groups reported several methods [6] to introduce the high absorption compounds including such as hafnium and zinc [79], tin-oxide [10,11], and tellurium [12] in base resin. In order to evaluate the effect of the high absorption material, it is important to measure the EUV absorption accurately. We have developed the EUVabsorption-coefficient measurement methods in high precision.

The EUV absorption coefficient is calculated using the following equation (1) [13].

$$
\mu=\frac{1}{D} \ln \left(\frac{1}{T}\right)
$$

Where $\mu, D$ and $T$ indicate the absorption coefficient, the resist thickness and the transmittance of the 
resist, respectively. For the accurate measurement of the EUV absorption coefficient, the equation (1) requires accurate measurement of both the resist thickness and EUV transmittance.

We have developed to measure the transmittance of EUV resist at BL 10 beamline [14-16] in the NewSUBARU synchrotron light facility [17] previously. In the past, we measured EUV transmittance by coating resist on a silicon-nitride freestanding membrane before and after measuring the intensity of EUV light transmitting the membrane. However, the accuracy of the transmittance measurement was not good. It was very hard to coat resist uniformly on the membrane, because the membrane was very thin and easy to deform during the spin coating.

Thus, we have developed the EUV absorption coefficient method employing direct resist-coating on a photodiode employed as a substrate. A photodiode has large sensing area of $10 \mathrm{~mm} \times 10$ $\mathrm{mm}$ and it is large enough to measure the EUV light intensity of the EUV beam size of $0.8 \mathrm{~mm}(\mathrm{H}) \times 0.1$ $\mathrm{mm}(\mathrm{V})$, this EUV light area size is twenty-five times larger than that of the silicon-nitride freestanding membrane. In addition, it is possible to coat the resist in high uniformity, because the surface of a photodiode is flat. Thus, it is practicable to measure transmittance accurately.

In this paper, in order to evaluate the EUV transmittance measurement accuracy, we measured the EUV absorption coefficient for various thickness of the resist.

\section{Experimental}

\subsection{Photodiode}

In previous paper, the SXUV-100 [18] (Opto Diode Corp.) photodiode was employed. The light sensing area size was $10 \mathrm{~mm} \times 10 \mathrm{~mm}$. This photo diode is a standard photodiode to measure soft Xray and hard X-ray light with high irradiation durability. However, this photodiode is much expensive. Thus, in this paper, we employed the S8552 (Hamamatsu Photonics K.K.) photodiode, which is normally used for UV and visible light intensity measurement and not so expensive to use in this absorption coefficient measurement method. Figure 1 shows the photograph of this photodiode. This photodiode has approximately 70-nm-thick oxidized on a surface. At first, the EUV spectral responsivity of EUV light for this photodiode was evaluated. Figure 2 shows the measurement result of EUV spectral responsivity of the SXUV-100 and S8552. The sensitivity of the SXUV-100 photodiode was already calibrated at Physikalisch-Technische Bundesanstalt (PTB). The S8552 photodiode had $1 / 3$ sensitivity of the SXUV-100 photodiode, which has enough sensitivity for the EUV transmittance measurement.

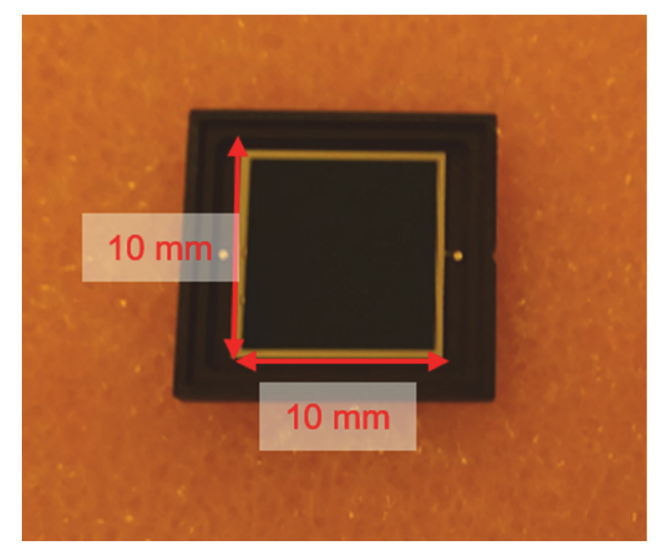

Fig. 1. Photograph of the S8552 photodiode.

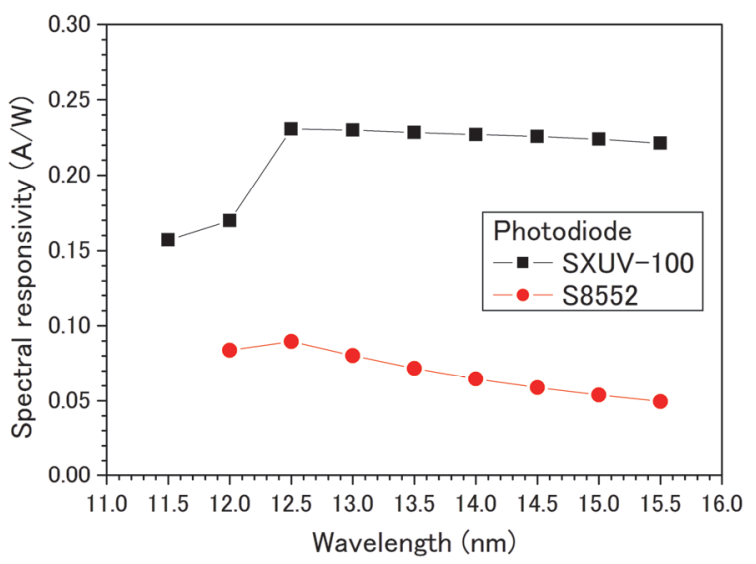

Fig. 2. EUV spectral responsivity of the S8552 and SXUV-100 photodiodes.

\subsection{Transmittance measurement method}

In this study, the EUV mask reflectometer [17] was used for the EUV transmittance measurement, which was located in BL 10 beamline at NewSUBARU synchrotron light facility. The light source of the BL 10 beamline employed white light generated from a bending magnet. And the EUV light is monochramated using the Monk-Gillieson type monochromator $[19,20]$ with a plane variedline-spacing grating (VLSG).

Furthermore, the energy resolution in EUV energy region was approximately $1300(\Delta \mathrm{E} / \mathrm{E})$ in this experiment. The exposure beam size of this EUV mask reflectometer was approximately 0.8 $\mathrm{mm}(\mathrm{H}) \times 0.1 \mathrm{~mm}(\mathrm{~V})$ in FWHM. The exposure wavelength was $13.5 \mathrm{~nm}$. Since the sensing area size of photodiode was much larger than that of the EUV 
light, the EUV light alignment to the sensing area of the photodiode is simple and easy.

Figure 3 shows a schematic diagram of the measurement method of a EUV resist transmittance. First, the photodiode current $I_{0}$ along the vertical at the center region was measured before the resist coating and then the photodiode current $I$ was measured after the resist coating. The transmittance $T$ was calculated the ratio of $I$ and $I_{0}$. The transmittance $T$ can describe by the following equation (2),

$$
T=\frac{I}{I_{0}} .
$$

Since the photodiode is taken out from the reflectometer after the measurement before coating, it takes a few hours or more to measure the transmittance after the coating. During this time interval, the EUV light intensity varies within $1 \%$. For the accurate transmittance measurement, the EUV light intensity normalized with the calibrated photodiode at PTB is used.

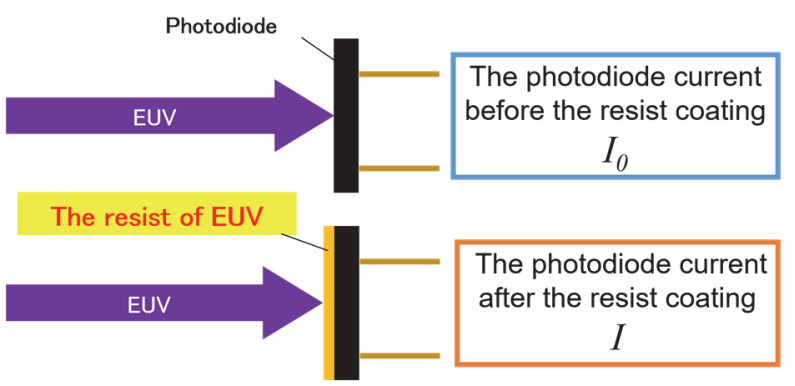

Fig. 3. Schematic diagram of the EUV transmittance measurement method.

\subsection{Thickness measurement method}

A sample resist at this experiment employed ZEP520A (Zeon Corp.) which is non-chemicalamplified positive-tone resist and commonly used in electron-beam lithography. The resist thickness varied such as $25,50,75$, and $100 \mathrm{~nm}$. This resist processing requires the pre-bake to evaporate the solvent and having adhesion to a substrate. The bake temperature of the ZEP520A resist was $180^{\circ} \mathrm{C}$. The resist coated on the photodiode was baked by an oven for one hour. In order to evaluate the heating damage of the photodiode, the EUV light intensity was measured before and after the baking the photodiode with non-resist coating at the resist prebake condition of $200^{\circ} \mathrm{C}$ in temperature at one hour. Figure 4 shows results of the EUV spectra responsivity before and after baking. The EUV spectra responsivity did not changed between before and after baking. Therefore, the baking process could not affect the EUV photodiode sensitivity.

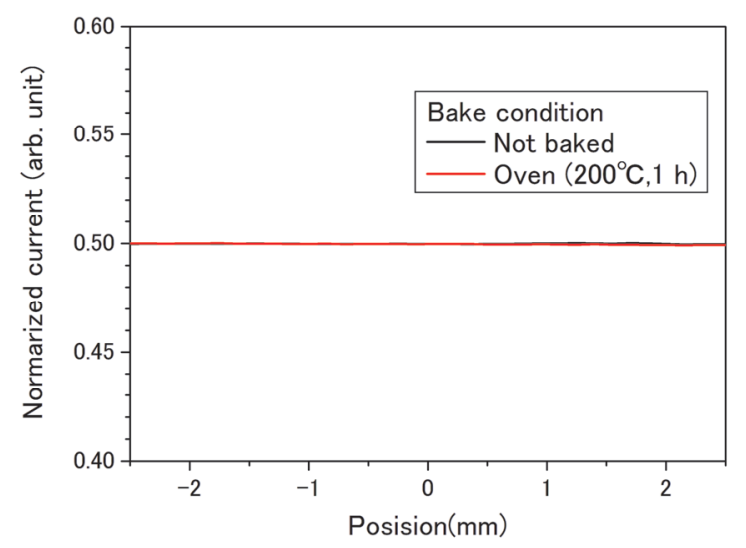

Fig. 4. Photodiode-heating-damage evaluation result of before and after baking at the temperature of $200^{\circ} \mathrm{C}$ using an oven. The photodiode current ratio under the EUV irradiation before and after heating are shown.

In previous study, the resist thickness was measured using a non-contact spectroscopic reflectometer of NanoSpec6100 (Nanometrics Inc.). The measurement spot size is $25 \mu \mathrm{m}$ in diameter, which is suitable for evaluate thickness distribution. This method needs accurate optical index of the resist and the substrate of the photodiode. We assume the photodiode substrate as $\mathrm{SiO}_{2}$ material. However, accurate structure of the photodiode was unknown. This caused resist thickness measurement error as discussed in Section 3.2.

In this study, the resist thickness was measured by $\mathrm{x}$-ray reflectometry (XRR), because we estimate that the resist thickness measurement accuracy is not good to use Nanometrics' thickness measurement system for the photodiode as a substrate which has several unknown layer. X'Pert Pro MRD (Panalytical) XRR system was used, which employs an X-ray tube with a $\mathrm{Cu}$ anode. The X-ray energy is approximately $8 \mathrm{keV}$. The XRR system measured reflectance spectrum of grazing angle $(\theta)$ from $0^{\circ}$ to $2^{\circ}$. The thickness of the resist could be measured by the interference fringe in the reflection. In the XRR measurement, the reflection fringe pitch depends on the film thickness and density of the film. The density is estimated by the total reflection angle. As a result, the film thickness can be measured independently of the chemical composition of the film. The evaluation area size is 
$10 \mathrm{~mm}$ in vertical direction, and $(0.1 / \sin \theta) \mathrm{mm}$ in horizontal direction, where $\theta$ is incident angle. Thus, the resist-thickness measurement value indicates the averaged thickness. However, the ceramic package edge of the photodiode was taller than the photodiode surface as shown in Figure 5. This edge blocks the incident $\mathrm{x}$-ray in XRR measurement. Thus, the package edge of photodiode for XRR measurement was removed.

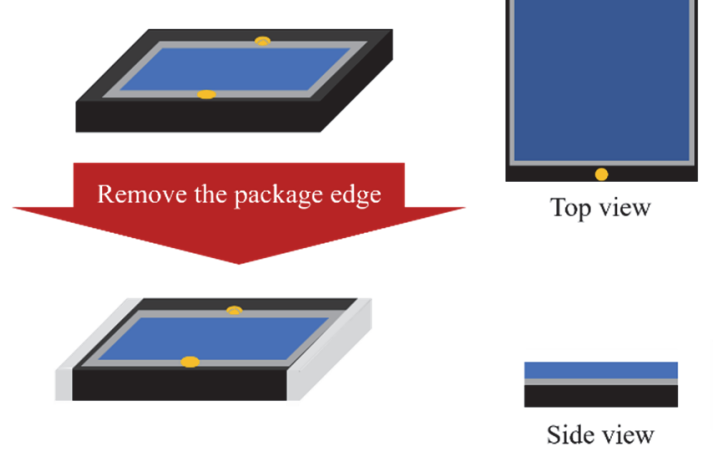

Fig. 5. Scheme of measurement pretreatment.

\section{Result and discussion}

\subsection{The resist transmittance}

Figure 6 shows measurement results of the resist transmittance of 4 samples which has four kinds of the resist thickness and the averaged resist transmittances of each sample were 89.1, 79.5, 74.9, and $65.6 \%$. Figure 7 is obtained by normalizing the transmittance with the average value obtained in Fig. 6 , and there is no variation within $5 \mathrm{~mm}$ as shown in Fig. 7. The transmittance uniformity was less than $0.6 \%$. This result indicates that the resist sample was coated with good uniformity on the surface of each photodiode.

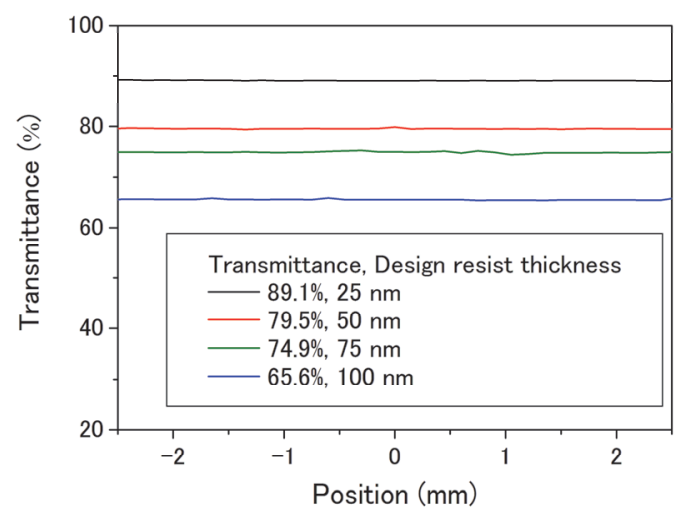

Fig. 6. Measurement results of EUV transmittance of ZEP520A resist.

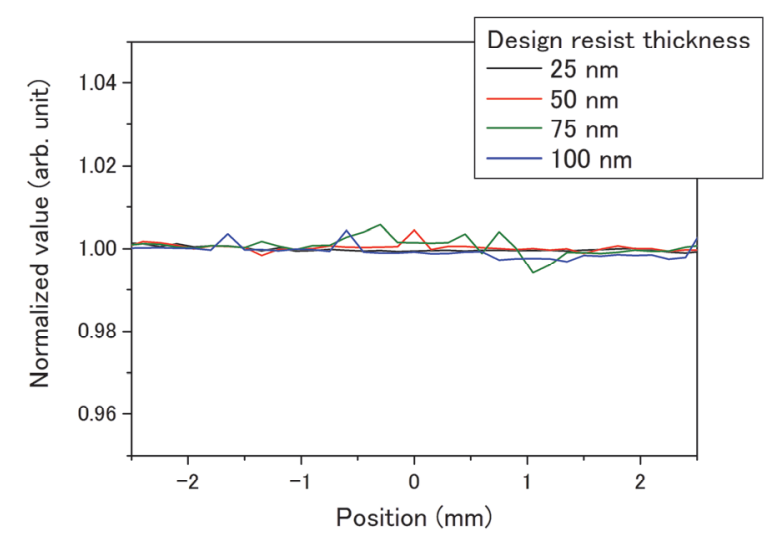

Fig. 7. Normalized transmittance by the average resist transmittance.

\subsection{The resist thickness}

Figure 8 shows the reflection spectrum measured by XRR. Before the resist coating, there was no fringe structure. It indicates that there was no thinlayer structure on the photodiode. The fringe structures were clearly observed after the resist coating on photodiode, and its fringe period depends on the resist thickness. The thicknesses were evaluated by the fringe period as listed on Table 1 .

Table 1. Results of resist thickness measurement in two methods.

\begin{tabular}{|l|c|c|c|c|}
\hline Sample \# & $\# 1$ & $\# 2$ & $\# 3$ & $\# 4$ \\
\hline Design resist thickness (nm) & 25 & 50 & 75 & 100 \\
\hline $\begin{array}{l}\text { Averaged resist thickness } \\
\text { (NanoSpec6100) (nm) }\end{array}$ & 25.5 & 49.7 & 62.7 & 108.1 \\
\hline $\begin{array}{l}\text { Peak to valley range of the } \\
\text { thickness }\end{array}$ & $\leq 6 \%$ & $\leq 3 \%$ & $\leq 2 \%$ & $\leq 2 \%$ \\
\hline Resist thickness (XRR) (nm) & 33.1 & 61.8 & 76.5 & 109.3 \\
\hline
\end{tabular}




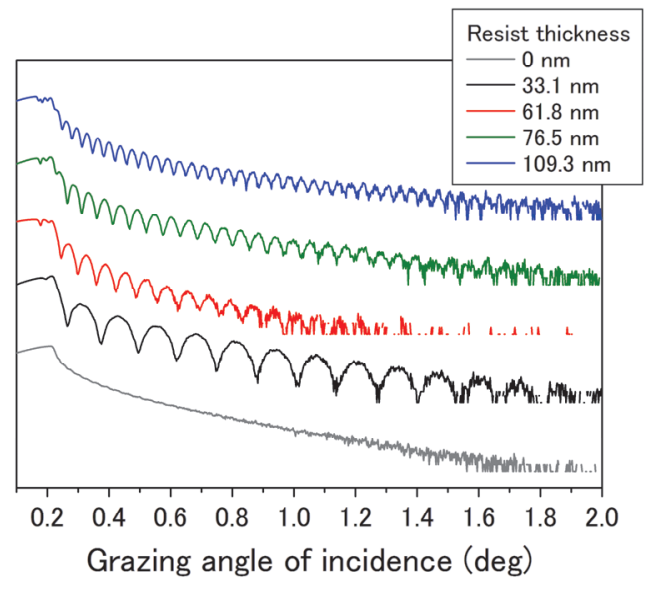

Fig. 8. The results of XRR measurement of resist on the photodiodes.

In another thickness measurement method, the resist thickness measurement on photodiode was carried out using Nanospec6100. The thickness measurement results between in two methods are shown in Table 1.

In addition, the thicknesses of resists on Si wafer substrates were measured by both methods. The relation of the resist thickness result in the two method is shown in Fig. 9. The thickness ratio between NanoSpec6100 and XRR with the Si waver substrate was approximately 0.95 for five thickness conditions. This shows the structure model of the $\mathrm{Si}$ wafer substrate using the Nanospec6100 was appropriate for the thickness measurement. However, at the photodiode sample, the thickness ratio was not same for the four thickness conditions. This result indicated the structure model of the photodiode was not appropriate, which caused large measurement error.

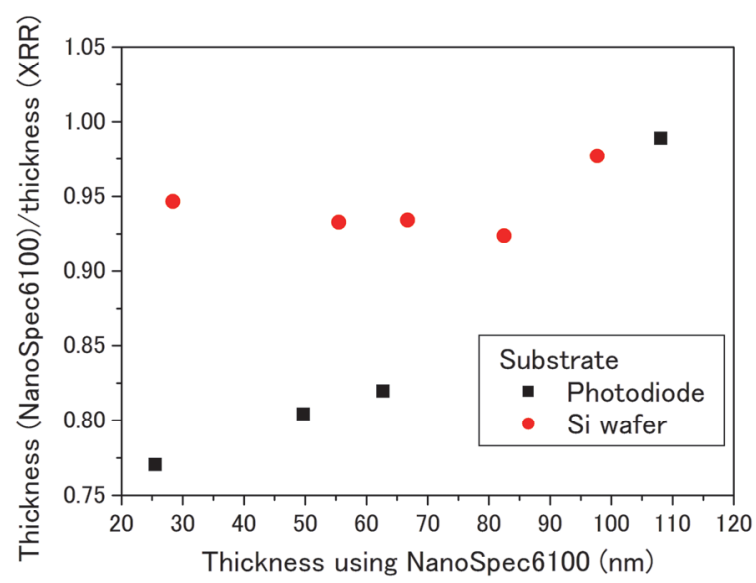

Fig. 9. Correlation of result thickness with two method.

\subsection{The resist EUV absorption coefficient}

Figure 10 shows the absorption coefficient computed with the transmittance and thickness measurement results by both NanoSpec6100 and XRR methods. The error bar indicates the $1 \%$ transmittance measurement error. The absorption coefficient using NanoSpec6100 result was not reliable, because that of \#4 sample was quite different from other samples. This error was due to the inappropriate optical structural model of the photodiode which has unknown layer structures.

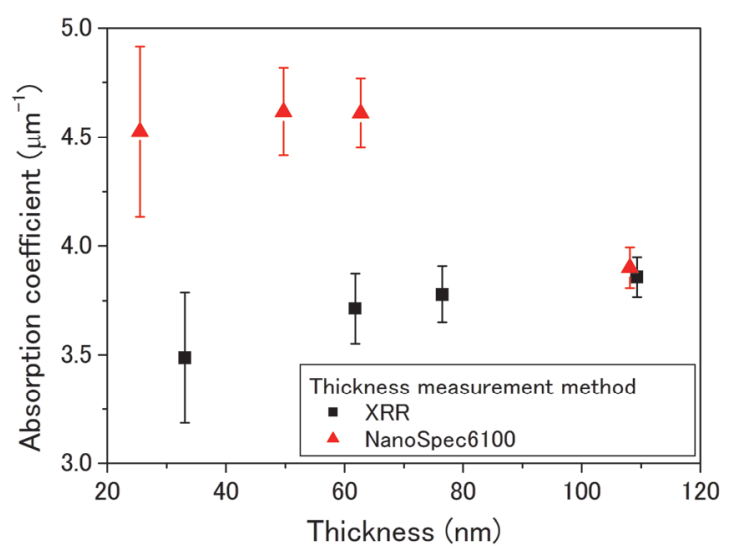

Fig. 10. Absorption coefficient results in two methods of resist thickness measurement.

The values of the absorption coefficient obtained from the four film thicknesses measured by XRR are all within the measurement error range. As a result, the absorption coefficient was measured accurately with combining the photodiode substrate and XRR thickness measurement method. Since the error bar was minimized with the thickest resist sample, the sample resist thickness should be thick for accurate absorption evaluation in the photodiode method. The calculated absorption coefficient of this resist take in account of the resist composition and the density of $1.3 \mathrm{~g} / \mathrm{cm}^{2}$ was approximately 4.6 and this value is quite different of our result of 3.8. This resist was very old one of 20 years ago, which would be decomposited at the $\mathrm{Cl}$ containing unit. In the future, we will evaluate this method using the simple sample such as PMMA.

\section{Conclusion}

We have developed the accurate absorptioncoefficient measurement method using the directresist coating on a photodiode. The XRR was employed in accurate resist thickness measurement on the photodiode. The reliable absorption coefficient was evaluated with four resist thickness condition. This accurate absorption measurement 
method will help the development of high absorption resist such as metal resist.

In near future, we will develop resist cleaning method on the photodiode without sensitivity degradation to achieve reuse of photodiode to save the thickness measurement cost.

\section{References}

1. H. Kinoshita, K. Kurihama, Y. Ishii, and Y. Torii, J. Vac. Sci. Technol., B7 (1989) 1648.

2. D. V. Steenwinckel, J. H. Lammers, L. H. A. Leunissen, and J. A. J. M. Kwinten, Proc. SPIE, 5753 (2005) 269.

3. M. Neisser and S. Wurm, Adv. Opt. Technol., 4 (2015) 235.

4. R. Peeters, S. Lok, J. Mallman, M. Noordenbur, N. Harned, P. Kuerz, M. Lowisch, E. Setten, G. Schiffelers, A. Pirati, J. Stoeldraijer, D. Brandt, N. Farrar, I. Fomenkov, H. Boom, H. Meiling, and Ron Kool, Proc. SPIE, 9048 (2014) 90481J.

5. A. Pirati, R. Peeters, D. Smith, S. Lok, A. Minnaert, M. Noordenburg, J. Mallmann, N. Harned, J. Stoeldraijer, C. Wagner, C. Zoldesi, E. Setten, J. Finders, K. Peuter, C. Ruijter, M. Popadic, R. Huang, M. Lin, F. Chuang, R. Es, M. Beckers, D. Brandt, N. Farrar, A. Schafgans, D. Brown, H. Boom, H. Meiling, and R. Kool, Proc. SPIE, 9422 (2015) 94221P.

6. H. Nakagawa, T. Naruoka, and T. Nagai, $J$. Photopolym. Sci. Technol., 27 (2014) 739.

7. A. Grenville, J. T. Anderson, B. L. Clark, P. D. Schepper, J. Edson, M. Greer, K. Jiang, M. Kocsis, S. T. Meyers, J. K. Stowers, A. J. Telecky, D. D. Simone, and G. Vandenberghe, Proc. SPIE, 9425 (2015) 94250S.

8. J. Stowers, J. Anderson, B. Cardineau, B. Cleark, P. D. Schepper, J. Edson, M. Greer, K. Jiang, M. Kocsis, S. Meyers, A. Telecky, A.
Greenville, D. De Simone, W. Gillijns, and G. Vandenbergle, Proc. SPIE, 9779 (2016) 977904.

9. D. De Simone, M. Mao, M. Kocsis, P. D. Schepper, F. Lazzarino, G. Vandenberghe, J. Stowers, S. Meyers, B. L. Clark, A. Grenville, V. Luong, F. Yamashita, and D. Parnell, Proc. SPIE, 9776 (2016) 97760B.

10. B. Cardinean, R. D. Re, H. A. Mashat, M. Marnell, M. Vockenhuber, Y. Ekinci, C. Sarma, M. Neisser, D. A. Freedman, and R. L. Bainarad, Proc. SPIE, 9051 (2014) 90511B.

11. E. Buitrago, R. Fallica, D. Fan, T. S. Kulmala, M. Vockenhuber, and Y. Ekinci, Microelectron. Eng., 155 (2016) 44.

12. H. Kudo, H. Ishihara, and M. Tomita, Chem. Lett., 40 (2011) 762.

13. A. Sekiguchi, Y. Matsumoto, T. Harada, T. Watanabe, and H. Kinoshita, Proc. SPIE, 9422 (2015) 94222L.

14. Y. Fukushima, T. Watanabe, T. Harada, and H. Kinoshita, J. Photopolym. Sci. Technol., 22 (2009) 85.

15. R. Ohnishi, T. Watanabe, Y. Fukushima, T. Harada, and H. Kinoshita, Jpn. J. Appl. Phys., 48 (2009) 06FA08.

16. D. Mamezaki, M. Watanabe, T. Harada, and T. Watanabe, J. Photopolym. Sci. Technol., 29 (2016) 749.

17. M. Kuki, T. Uemura, M. Yamaguchi, T. Harada, T. Watanabe, Y. Muramatsu, and H. Kinoshita, J. Photopolym. Sci. Technol., 28 (2015) 531.

18. R. Korde, C. Prince, D. Cunningham, R. E. Vest, and E. Gullikson, Metrologia, 40 (2003) S145.

19. M. C. Hettrick and J. H. Underwood, AIP Conf. Proc., 147 (1986) 237.

20. M. Koike and T. Namioka, Appl. Opt., 36 (1997) 6308. 